\title{
Erratum to: Ureaplasma diversum as a cause of pustular vulvovaginitis in bovine females in Vale Guapore, Mato Grosso State, Brazil
}

\author{
João Guilherme L. N. Gaeti • Marconni V. C. Lana • Gustavo S. Silva • Leticya L. Lopes • \\ Camila G. de Campos • Fernanda H. Maruyama • Edson M. Colodel • Eduardo F. Costa • \\ Luis G. Corbellini • Luciano Nakazato • Caroline A. Pescador
}

Published online: 28 June 2014

(C) Springer Science+Business Media Dordrecht 2014

\section{Erratum to: Trop Anim Health Prod \\ DOI 10.1007/s11250-014-0614-5}

There is an error in relation to the authors name and the institution which they belong. Here is the correct way:

João Guilherme L. N. Gaeti, Marconni V. C. Lana, Gustavo S. Silva, Leticya L. Lopes, Camila G. de Campos, Fernanda H. Maruyama, Edson M. Colodel, Eduardo F. Costa, Luis G. Corbellini, Luciano Nakazato, Caroline A. Pescador

J.G.L.N. Gaeti, M.V.C.Lana, G.S.Silva, L.L. Lopes, C.G.Campos, E.M. Colodel, C.A. Pescador

Veterinary Pathology Laboratory, Federal University of Mato Grosso (UFMT), Av. Fernando Corrêa da Costa, 2367, Bairro Boa Esperança, Cuiabá, Mato Grosso CEP 78060-900, Brazil
E. F. Costa, L. G. Corbellini Epidemiology Laboratory, Federal University of Rio Grande do Sul (UFRGS), Av. Bento Gonçalves 9090, Bairro Agronomia, Porto Alegre CEP 51900-000, Brazil

\section{F. H. Maruyama, L. Nakazato}

Laboratory of Veterinary Molecular Biology, Federal University of Mato Grosso (UFMT), Av. Fernando Corrêa da Costa, 2367, Bairro Boa Esperança, Cuiabá, Mato Grosso CEP 78060-900, Brazil

The online version of the original article can be found at http://dx.doi.org/ 10.1007/s11250-014-0614-5.

J. G. L. N. Gaeti · M. V. C. Lana · G. S. Silva • L. L. Lopes •

C. G. de Campos • E. M. Colodel $\cdot$ C. A. Pescador $(\varangle)$

Veterinary Pathology Laboratory, Federal University of Mato Grosso

(UFMT), Av. Fernando Corrêa da Costa, 2367, Bairro Boa

Esperança, Cuiabá, Mato Grosso CEP 78060-900, Brazil

e-mail: carolpescador@yahoo.com.br

E. F. Costa $\cdot$ L. G. Corbellini

Epidemiology Laboratory, Federal University of Rio Grande do Sul

(UFRGS), Av. Bento Gonçalves 9090, Bairro Agronomia, Porto

Alegre CEP 51900-000, Brazil

F. H. Maruyama $\cdot$ L. Nakazato

Laboratory of Veterinary Molecular Biology, Federal University of Mato Grosso (UFMT), Av. Fernando Corrêa da Costa, 2367, Bairro Boa Esperança, Cuiabá, Mato Grosso CEP 78060-900, Brazil 